Fig. $2(a), F_{1}$ region. The ionization on an average diminishes for all the nights except for that of November $15-16$.

Fig. $2(b), F_{2}$ region. The ionization on an average diminishes for all the nights.

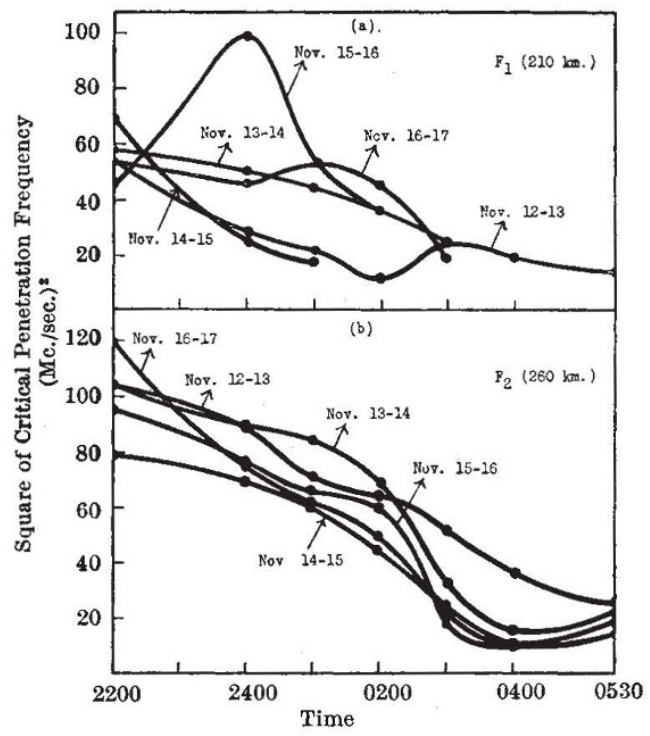

Fig. 2.

The increases in the ionization of the $E_{1}$ and $E_{2}$ regions (Fig. 1) on the night of November 14-15 are undoubtedly due to the impact of meteors. There was no other disturbing factor in this night. There might be some uncertainty with regard to the increases on the night of November $15-16$ in $E_{1}, E_{2}$ and $F_{1}$ ionization, as there was a magnetic storm in progress during that night. Since, however, the time of increase in the ionization of $E_{1}$ and $E_{2}$ on this night corresponds, as on the previous night, to the hour of maximum intensity of the Leonid shower, and also since no significant correlation between a magnetic storm and abnormal increase of the ionization of these regions has, as yet, been found in our latitude, it may safely be inferred that these increases are also due to the meteoric shower. With regard to the increase in the $F_{1}$ ionization during the same night, it is very doubtful if meteors were responsible for this, since the time of increase does not correspond with the time of maximum shower. Further, as in the case of the two $E$ regions, there was no increase of a similar nature on the previous night.

Since the heights of most frequent appearance and disappearance of meteors, visible to the naked eye, lie between $160 \mathrm{~km}$. and $70 \mathrm{~km}$., it is to be expected that any increase of ionization caused by the meteors should be confined within these limits. This is what has actually been observed. Though, in the course of our observations, we were able to detect each of the four distinct regions of maximum ionization, it was only the lower ones- $E_{1}$ and $E_{2}-$ which were found to be affected by the meteoric shower. The fact that these regions showed increase separately and were not merged into one continuous region due to meteoric impact —as, at first thought, might have been expected - also receives an easy explanation if we remember that a region which is already ionized should undergo proportionately greater increase of ionization than a region which is not originally ionized.
An account of this work with fuller details will appear shortly in the Indian Journal of Physics. The author is indebted to Prof. S. K. Mitra and Dr. H. Rakshit for their kind interest in the work.

Wireless Laboratory,

University College of Science,

92 Upper Circular Road, Calcutta.

Jan. 18.

${ }^{1}$ Skellet, A. M., Proc. Inst. Rad. Eng., 28, 132 (1935).

${ }^{2}$ Mitra, S. K., Syam, P., and Ghose, B. N., NAture, 133, 533 (1933).

\section{Figures of the Earth and Moon}

A RECENT paper of mine, noticed in NATURE for February 27, p. 381, needs a correction, since the next issue of the Monthly Notices of the Royal Astronomical Society (p. 126) contains a correction by Prof. E. W. Brown to the calculated motions of the moon's perigee and node, which had stood for about twenty years. The change for the perigee is about three times what appeared to be the probable error of the previous value. Brown, in a letter to me, has queried the probable errors that I used; but provisionally retaining them until better estimates are available, I have made a new solution, which is : $J=0.0016361 \pm 0.0000059 ; 1 / e=297.19 \pm 0.52$; $J^{\prime}=0.000316 \pm 0.000024 ; K^{\prime}=0.000115 \pm$ $0.000048 ; f=0 \cdot 69 \pm 0.06$.

The ellipticity of the earth is only slightly affected; the chief change is in the ellipticity of the moon's equator, which is proportional to $K^{\prime}$ and is more than doubled. It is now almost midway between the two inconsistent values of Hayn and Stratton, and affords no ground for preferring either to the other. The coefficient of $g_{0} \sin ^{2} \dot{\varphi}$ in gravity will be increased to $0 \cdot 0052911 \pm 0 \cdot 0000059$.

The need for a modern series of observations to determine the moon's librations in longitude is clear ; in the above determination, $K^{\prime}$ rests entirely on the motion of the perigee. If it is correct, the period of the free libration in longitude should be close to 50 sidereal months and the amplitude of the annual forced libration $0 \cdot 84^{\prime}$.

\section{St. John's College, Cambridge.} HAROLD JEFFReYS

\section{Planetary Positions at Sunset in 1937}

The hour after sunset is possibly the time most often chosen by many for noticing the bright planets, chiefly because Venus and Mercury scarcely let us notice them at any other time, except by insisting on inconvenient wakefulness before sunrise.

In the accompanying drawing (Fig. 1), and for the English sunset sky, the positions of the sun and the bright planets are indicated for the first day of every month of the year. Number 1 stands for the first day of January, 2 for the first of February, and so on, 13 standing for the first of January 1938. The positions of the setting sun on the first day of two months like January and December are not really the same, but they are sufficiently near to one point to be marked the same for the purposes of this diagram.

The positions of Mercury lie on a peculiarly tangled path, and the middle of April (the fourth month) 